\title{
Reflexive Games in Management
}

\section{Andrew Schumann}

University of Information Technology and Management

in Rzeszow, Poland

e-mail: andrew.schumann@gmail.com

\begin{abstract}
:
In this paper reflexive games are defined as a way to act beyond equilibria to control our opponents by our hiding motives. The task of a reflexive game is to have the opponent's actions become transparent for us, while our actions remain obscure for the competitor. In case a reflexive game is carried out between agents belonging to the same organisation (corporation, company, institute), success in a reflexive game can be reached by a purposeful modification of some components of a controlled system. Such a modification for the guaranteed victory in a reflexive game is called reflexive management. This kind of management uses reflexive games to control a knowledge structure of agents in a way their actions unconsciously satisfy the centre's goals.

Keywords: reflexive game, reflexive management, speech competence, discourse community.
\end{abstract}

\section{Introduction}

One of the directions in pragmatic studies is presented by reflexive games. For the first time, Vladimir Lefebvre formulated reflexive games assuming many reflexion levels [8], [9], [10]. Reflexive games are understood as an extension of epistemic games [2]. The game-theoretic mathematics for the early ideas of Lefebvre has been developed by Dmitry Novikov and Aleksandr Chkhartishvili [3], [4], [5], [6]. In this paper I appeal to the approach to reflexive games proposed in [12], [13], [14]. This approach is unconventional and assumes cellular-automatic calculations. First, I define the context of reflexive games (section 2) and show why in reflexive games there are no conventional equilibria. Then I introduce the notion of reflexive games in accordance with the ideas of [12], [13], [14] (section 3). Further, I show how we can apply reflexive games in the management practice within the so-called reflexive management (section 4). Finally, I consider the role of reflexive management in discourse communities (section 5).

\section{Enemies and Games Beyond Nash Equilibria}

In the Austrian school of economics it is supposed that the simple mutually advantageous interchange is always possible. In the words of game theorists, this means that the Nash equilibrium is always possible. For example, I produce apples, my neighbour produces pears. Nevertheless, I 
like pears and my neighbour apples. Then the Nash equilibrium is reached by the mutually advantageous interchange of apples and pears, for example by using the formula: one apple for one pear and vice versa. During the interchange each actor is rational, knows the set of game players, the goal functions and the admissible set of actions of all players, and also knows the set of possible values of states of affairs. Such knowledge can be reached, in particular by a public communication of appropriate information of all agents met at one place. This communication allows them to find the Nash equilibrium, a simple mutually advantageous interchange. In the Nash equilibrium there is a parity of reflexive relations of all players. On the one hand, both actors have autonomy, different goal functions and, on the other hand, both help each other to reach goals by means of a mutually advantageous interchange, knowing everything about each other. As the first approximation, the stock exchange is an example of such an equilibrium.

Let us suppose now that rational agents are our enemies. They do not wish to help us to reach the equilibrium of our goal functions by means of an interchange. In every possible way they hinder us from having the usual interchange with other players (for example, they use dumping practices so that we will go bankrupt). In this case the Nash equilibrium cannot be reached. We cannot wait for a simple mutually advantageous interchange of goods.

Competitiveness complicates any strategy of reaching a maximal guaranteed payoff. We should already deal with reflexive games in order to evaluate other actors, for example, to reconstruct their goal functions taking into account circumstances in which they can try to delude their environment concerning the original motives of their acts. The main task of reflexive games is to hide true motives and goals, not to be transparent for others, but to know everything important about them. Let us consider Edgar Allen Poe's example of reflexive games:

I knew one [schoolboy] about eight years of age, whose success at guessing in the game of 'even and odd' attracted universal admiration. This game is simple, and is played with marbles. One player holds in his hand a number of these toys, and demands of another whether that number is even or odd. If the guess is right, the guesser wins one; if wrong, he loses one. The boy to whom I allude won all the marbles of the school. Of course he had some principle of guessing; and this lay in mere observation and measurement of the astuteness of his opponents. For example, an arrant simpleton is his opponent, and, holding up his closed hand, asks, 'are they even or odd?' Our schoolboy replies, 'odd,' and loses; but upon the second trial he wins, for he then says to himself, 'the simpleton had them even upon the first trial, and his amount of cunning is just sufficient to make him have them odd upon the second; I will therefore guess odd;'-he guesses odd, and wins. Now, with a simpleton a degree above the first, he would have reasoned thus: 'This fellow finds that in the first instance I guessed odd, and, in the second, he will propose to himself, upon the first impulse, a simple variation from even to odd, as did the first simpleton; but then a second thought will suggest that this is too simple a variation, and finally he will decide upon putting it even as before. I will therefore guess even;'- - he guesses even, and wins. Now this mode of reasoning in the schoolboy, whom his fellows termed 'lucky,'-what, in its last analysis, is it?

'It is merely,' I said, 'an identification of the reasoner's intellect with that of his opponent.'

'It is,' said Dupin; and, upon inquiring, of the boy by what means he effected the thorough identification in which his success consisted, I received answer as follows: 'When I wish to find out how wise, or how stupid, or how good, or how wicked is any one, or what are his thoughts at the moment, I fashion the expression of my face, as accurately as possible, in accordance with the expression of his, and then wait to see what thoughts or sentiments arise in my mind or heart, as if to match or correspond with the expression.' This response of the schoolboy lies at the bottom of all the spurious profundity which has been attributed to Rochefoucault, to La Bougève, to Machiavelli, and to Campanella (Edgar Allen Poe, The Purloined Letter). 
In this example, the schoolboy has success at guessing in the game of 'even and odd,' because he considers it not as simple guessing, but as a reflexive game and correctly defines two kinds of gamers: 'an arrant simpleton' who permanently changes the strategy upon different trials, and a 'simpleton a degree above the first' who uses the same strategy upon different trials for cheating (cheating since the game is understood by gamers as pure guessing). In other words, the game of 'even and odd' assumes two levels of reflexion: the first level consisting in using casually different strategies, the second consisting in using the same strategies and in avoiding casual choices of strategies. Different people with different intelligent abilities play at different reflexive levels. How many levels can exist in reflexive games in all?

Let us imagine a nightmare. A huge monster runs after us and its speed is obviously faster. We face two caves. The monster does not have time to see which of the caves we choose. The first cave is twisting and the second is a straight line as a tunnel. It is an example of a reflexive game. I select a cave, assuming which cave the monster will choose. Let us consider the possible levels of reflexion:

- The reflexion of zero level: I do not think that the animal thinks, and the animal does not think that I think. I choose the twisting cave, my arguments are as follows: in the twisting cave any speed is reduced and I have a possibility to escape from the monster; in running through it I will not be in the monster's sight and my further actions will not be known by the animal. For the monster the reflexion of zero level can mean a choice of the direct cave, as it is easier to run through this cave.

- The first-level reflexion: I think that the animal thinks, and the animal thinks that I think. Formally: Think ${ }_{A}\left(\operatorname{Think}_{B}\right)$ and $\operatorname{Think}_{B}\left(\operatorname{Think}_{A}\right)$, where agent $A$ is me and agent $B$ is the monster. The monster at this level of reflexion will run through the twisting cave. It already tries to predict my behaviour and my choice of cave. I also will run through the twisting cave, as I know that at the zero level of reflexion the animal chooses the direct cave.

- The second-level reflexion: I think that the animal thinks, thinking that I think, and the monster thinks that I think, thinking that the monster thinks. Formally: $\operatorname{Think}_{A}\left(\operatorname{Think}_{B}\left(\operatorname{Think}_{A}\right)\right)$ and $\operatorname{Think}_{B}\left(\operatorname{Think}_{A}\left(\operatorname{Think}_{B}\right)\right)$. Having selected the twisting path, I generally did not evaluate the mental abilities of the monster to deceive me. I assumed that it is able only to commit direct actions and is not able to deceive. However, this assumption can become false. The monster can predict my actions in order to understand what I think of it. The second level of reflexion is that I assume that the monster wishes and is able to predict my actions as an intelligent being. Then I should choose the direct cave. My arguments: any intelligent being selects the twisting cave, because it is easier to be rescued in it, but such logic is transparent for any rational agent, the same for the monster, if it is rational. At the second level of reflexion I try to predict the actions of the monster recognising that it considers me an intelligent being and I try to act not in the way it expects. The monster at the second level of reflexion also runs through the straight cave.

- The third-level reflexion: I think that the animal thinks, thinking that I think, thinking that I think. $\operatorname{Think}_{B}\left(\operatorname{Think}_{A}\left(\operatorname{Think}_{B}\left(\operatorname{Think}_{A}\right)\right)\right)$ and $\operatorname{Think}_{A}\left(\operatorname{Think}_{B}\left(\operatorname{Think}_{A}\left(\operatorname{Think}_{B}\right)\right)\right)$. At the second level of reflexion I detect the monster's ability (as a reflexive player of the first level) to predict my behaviour, but I have not yet assumed that the monster itself can have the ability of reflexion of the second level and it can act not in the way I expect. At the second level of reflexion I expected that it should run through the straight cave. Nevertheless, the animal can know itself about this by my waiting, therefore at the third level of reflexion I select the twisting cave. My arguments: any being capable of an elementary reflexion of the second level will select the direct cave, knowing that only the most naive rational agents select the twisting cave. We wish to act unpredictably for rational agents, therefore we choose again the twisting cave. But at the third level of reflexion the monster will run also through the twisting cave. It also assumes that we are capable of deceit.

- The fourth-level reflexion:

$\operatorname{Think}_{A}\left(\operatorname{Think}_{B}\left(\operatorname{Think}_{A}\left(\operatorname{Think}_{B}\left(\operatorname{Think}_{A}\right)\right)\right)\right), \operatorname{Think}_{B}\left(\operatorname{Think}_{A}\left(\operatorname{Think}_{B}\left(\operatorname{Think}_{A}\left(\operatorname{Think}_{B}\right)\right)\right)\right)$. 
However, my logic with the desire to be unpredictable can also be transparent for the monster. Consequently, I cannot be rescued again through the twisted cave. I should choose the direct cave. Which cave should I run through then? Which cave will the animal run through?

In this game of choosing the caves I lose and the monster wins, if $n>0$ and my level of reflexion and the monster's level of reflexion are the same number $n$. I win, if the monster's level of reflexion is $n$ and my level of reflexion is $n+1$.

This example with the monster shows that reflexion levels can be an arbitrary natural number. If I do not know the monster's mental abilities, I cannot select the level of reflexion upon which I should act. Then I will stand before both caves without the possibility of finding any true level of reflexion. In this time the monster will overtake me and eat me. It is an example of the reflexivity paradox, i.e. the impossibility of defining a true level of reflexion for a successful interaction with competitors.

Another example of a reflexive game when the reflexivity paradox is possible is hide-andseek. The first actor hides in one of several rooms with different lighting, and another agent should select that room where he will search for the first actor. The degree of lighting is known by both agents. The strategies of the agents are as follows. The second actor, who searches, rather prefers to search where it is lighter (easier to find). On the contrary, the first actor, who hides, rather prefers to hide in dark rooms, because there are more chances to be undiscovered. It is a zero level of reflexion for both agents. The increase of reflexion degrees means that it becomes clear to the agent that it is clear also to his opponent, etc. If I do not know the mental abilities of the opponent, the paradox of reflexivity will hold. Then I cannot select the rooms in which it is more preferable to search (hide). At the same time, the first actor, who hides, wins, if $n>0$ and his level of reflexion is $n+1$, when his opponent's level of reflexion is $n$. The second, who seeks, wins if $n>0$ and his level of reflexion, $n$, is the same as his opponent's level of reflexion.

It is obvious that if there are no data about a competitor's mental abilities at all, I can act at the zero level of reflexion, i.e. I can ignore the competitor's intellectual possibilities in his play against me. If there is an occasion to guess their mental abilities, I select reflexion level $n$ with respect to the opponent's abilities to play in reflexive games and my possibilities of winning.

If at least one agent selects a game strategy assuming a non-zero level of reflexion, then this game is called a reflexive game. Its essence consists in finding the level of reflexion $n$ of the competitor $(n>0)$ to move onto reflexion level $n$ (if I have advantages at the equal level of reflexivity) or $n+1$ (if I have no advantages) and to act on the basis of the given level. The task of a reflexive game is to have the opponent's actions become transparent for us, while our actions remain obscure for the competitor.

\section{On the Notion of Reflexive Game}

Let us notice that in our everyday life we permanently face reflexive games. Thereby gamers can follow different levels of reflexion upon different trials of the same game. A reflexion level is not constant. It is a dynamic index. Accordingly, the victory in a reflexive game is determined by who has managed in most cases to be in dialogues at a level of reflexion $n$ or $n+1$ while the interlocutor remained at level $n$. The more difficult the reflexive game, the more information we should give about ourselves to uncover all motivations and all predispositions of the interlocutor.

There are too many examples of daily reflexive games. Let us consider relationships in a family. Does a husband or a wife have a priority in a reflexive game? Who should be the leader in a family? Are equal relations possible? Or consider relationships with subordinates. Should reflexive games be carried out in relations with subordinates?

Rules in reflexive games depend on the following parameters:

- number of agents (a leader of a group is presented by an agent who is capable in dialogues of being at reflexion level $n$ or $n+1$ while all other interlocutors remain at level $n$ upon major trials of the same game; notice that for each pair of agents the number $n$ can be different); 
- preferences of agents (different goal functions and dependences of their payoff on actions, e.g. when we know that each agent is interested in a maximisation of payoff and for this purpose (s)he commits a minimal set of certain actions, and for different agents this set can be different);

- set of admissible actions of agents (there are actions which are unacceptable for all in the group of agents, and there are actions which are expected or not expected by other agents, but these actions are admissible for the entire group of agents);

- knowledge of agents (at the moment of decision making agents should be informed, probably falsely, about all preferences of other agents);

- order of moves (sequence of choices of actions, comprehensible to all in the group of agents).

Thus, preferences express what agents want, sets of admissible operations express what they can do, knowledge expresses what they know, and order of moves express when they select actions.

The larger the number of agents in a group, the more complex task to be a leader (to reach a victory in a reflexive game upon major trials). An elementary case is the game with two actors. Such games can be considered in the bimatrix form. So, the monster's run is a bimatrix reflexive game of the form $(x, y)$, where $x$ is my choice ( 0 is a straight line, 1 is a twisted cave), $y$ is the monster's choice ( 0 is a straight line, 1 is a twisted cave). I win, if $x \neq y$, and the monster wins, if $x=$ $y$. Values of $x$ and $y$ depend on the reflexion level. At an equal level of reflexion $x=y$ and the monster wins, as at the level of direct actions it has advantages. At reflexion level $n$ for the monster and $n+1$ for us there is no Nash equilibrium.

A classical example of a bimatrix reflexive game is the Prisoner's Dilemma. Each of two prisoners can choose one of the following two actions: "to confess a crime" and "not to confess a crime." If both agents cooperate with the police, both are sentenced and the vector (1 year, 1 year) is their payoff. If the first confesses and the second does not, then the first goes free and the second is sentenced and the vector (goes free, 3 years) is their payoff. If the second confesses and the first does not, then ( 3 years, goes free). And if both do not confess, their punishment will be equal ( 2 years, 2 years).

In reflexive games we deal with an unlimited hierarchy of cognitive pictures. Let us consider a bimatrix game with agents $i$ and $j$. Each of them can have their own picture about a state of affairs $A$. Denote these pictures by $K_{i} A$ and $K_{j} A$ respectively. The first-order reflexion (thoughts about pictures of the opponent) is expressed by means of pictures of the second order which are designated by $K_{j} K_{i} A$ and $K_{i} K_{j} A$ where $K_{j} K_{i} A$ are pictures of agent $j$ about pictures of agent $i, K_{i} K_{j} A$ are pictures of agent $i$ about pictures of agent $j$. The reflexion of the second order defines which pictures of one opponent are related to pictures of another opponent. At this level of reflexion pictures of the third order $K_{i} K_{j} K_{i} A$ and $K_{j} K_{i} K_{j} A$ are generated. And so ad infinitum. The collection of all pictures $K_{i} A, K_{j} A, K_{j} K_{i} A, K_{i} K_{j} A, K_{i} K_{j} K_{i} A, K_{j} K_{i} K_{j} A$ etc. makes an infinite hierarchy.

Definition 1. The reflexion of the agent $i$ on the $n$-th level in bimatrix games is expressed by $(n+1)$ order knowledge operators $K_{i}^{n+1} A=K_{i} K_{j} K_{i} \ldots A$, where on the right side there are $n+1 K_{m}$-operators $(m=i, j)$.

Let us consider two agents $i$ and $j$ and suppose that the reflexive game takes place on level $n$. This means that we have $K_{i}^{n+1} A$ and/or $K_{j}^{n+1} A$ which are understood as perlocutionary effects of illocutionary acts [15], [16] and satisfy requirements:

$$
\begin{gathered}
\left(K_{i} A \cap K_{i} B\right) \Rightarrow K_{i}(A \cap B) \\
K_{i}(A \cup B) \Rightarrow\left(K_{i} A \cup K_{i} B\right) ; \\
K_{i}(A \cup B)=\left(K_{i} A \cap K_{i} B\right) ; \\
A \subseteq B \Rightarrow K_{i} A \subseteq K_{i} B \\
48
\end{gathered}
$$




$$
\begin{gathered}
A \subseteq K_{i} A ; \\
K_{i} K_{i} A=K_{i} A .
\end{gathered}
$$

For more details see [12], [13], [14].

We know that $A \subseteq \ldots \subseteq K_{j}^{n} A \subseteq K_{i}^{n+1} A$ and $A \subseteq \ldots \subseteq K_{i}^{n} A \subseteq K_{j}^{n+1} A$. Therefore $K_{i}^{n+1} A \cap K_{j}^{n+1} A \neq \varnothing$.

Definition 2. The payoff of a reflexive game on the $n$-th level in accordance with $K_{i}^{n+1} A$ or $K_{j}^{n+1} A$ is called performative equilibrium of this game.

We have the following possibilities:

- both $K_{i}^{n+1} A$ and $K_{j}^{n+1} A$ are a performative equilibrium - this means that agents $i$ and $j$ are on the $n$-th level of reflexion, simultaneously;

- only $K_{i}^{n+1} A$ is a performative equilibrium (then we can take $K_{j}^{n+1} A=K_{j}^{n} A$ ) - this means that agent $i$ stays on the $n$-th level of reflexion, but agent $j$ stays on the $(n-1)$-th level of reflexion;

- only $K_{j}^{n+1} A$ is a performative equilibrium (then we can take $K_{i}^{n+1} A=K_{i}^{n} A$ ) - this means that agent $j$ stays on the $n$-th level of reflexion, but agent $i$ stays on the $(n-1)$-th level of reflexion.

For any reflexive game on the $n$-th level of reflexion we can build up a tree of graphs. Vertices of the tree correspond to real or phantom agents, participating in reflexive game. Branches of the tree simulate a mutual knowledge of agents on reflexion level $n$ : if from (real or phantom) agent $i$ there exists a path to agent $j$, then agent $j$ is correctly informed about agent $i$. In this case $K_{j}^{n+1} A$ is a performative equilibrium. If both $K_{i}^{n+1} A$ and $K_{j}^{n+1} A$ are a performative equilibrium of the same game, then an appropriate tree has a loop.

In a reflexive game on level $n$ it is important for agent $i$ that $K_{i}^{n} A \subseteq K_{j}^{n+1} A$ holds, because it means that agent $i$ has really corresponded to level $n$. Correctly defining the level $n$ of reflexion implies a victory in a game. Let us consider the game of two brokers to show how it is sophisticated sometimes to define $n$. Two brokers at a stock exchange have appropriate expert systems which have been used for the support of decision making. The network administrator illegally copied both expert systems and sold each broker an expert system of his opponent. Then he tries to sell each of them the following information: "Your opponent has your expert system." Then the administrator tries to sell the information: "Your opponent knows that you have his expert system," etc. How should brokers use the information received from the administrator and also what information on what iteration is essential? Theoretically, reflexive level $n$ can be any natural number.

\section{On the Notion of Reflexive Management}

Any everyday dialogue can be considered a reflexive game. Each person, speaking those or other things, tries to obtain something from us. We always try to understand the motives (s)he has for talking to us. Do they (s)he wish only to learn something from us or to influence us? How exclusive is the message which (s)he utters? Will we begin to know more on the topic after the talk? Is (s)he sincere? How sincerely does (s)he express for us his/her strategy of creative reasoning?

Emotions, which are expressed in illocutions, are one of the main forms of reflexion. The interchanging of emotions is always a reflexive game, a method of manipulation of others. The character played by Sharon Stone in Basic Instinct (the 1992 movie) shows reflexive abilities in emotional management. How transparent are her emotions? Are we capable of winning emotionally in games with her or at least of reaching an emotional consensus? Her emotions are not at all transparent for us as are the emotions of coaching trainers who better know strategies of management struggle and overcome us in any reflexive game.

Insufficient knowledge (lack of common knowledge $K_{i}^{n+1} A$ ) of agent $i$ on reflexion level $n$ leads to an actual vector of actions on reflexion level $n$ that can differ from a vector expected by agent $i$. For reaching a performative equilibrium it is expedient to follow the following assumptions: 
1. The finite number of real and phantom agents participate in a reflexive game.

2. Equally informed agents select identical actions according to reflexion level $n$.

3. The rational behaviour of agents consists in that each of them aspires to maximise a goal function by a choice of appropriate actions, predicting which actions other agents will choose as rational agents from the point of view of knowledge of reflexion level $n$ about other agents.

In case a reflexive game is carried out between agents belonging to the same organisation (corporation, company, institute), success in a reflexive game can be reached by a purposeful modification of some components of a controlled system. Such a modification for the guaranteed victory in a reflexive game is called reflexive management. The principal kinds of reflexive management are as follows:

- institutional management (modification of admissible sets of actions of all groups of agents);

- motivational management (modification of goal functions of concrete agents);

- informational management (modification of information which agents use in decision making).

Informational management refers to the following kinds:

- informational regulating (purposeful influence on information about states of affairs);

- expert management (purposeful influence on information about models of decision making);

- active prognosis (purposeful spread of information about future values of parameters depending on states of affairs and actions of actors).

The task of reflexive management is formulated as follows: a controlling organ creates a knowledge structure of agents in a way such that a performative equilibrium satisfies the centre's goals (maximally favourable for this centre.)

Management of an opponent's decision-making can be carried out by means of suggestions to him/her of some foundations from which (s)he could logically infer decisions favourable to us. Such a process of suggesting foundations for an opponent's decision-making is called reflexive management. Reflexive management can be performed by means of saying false information about a state of affairs (creation of false objects), by means of suggesting an opponent's purposes (provocations and intrigues, acts of terrorism and ideological diversions), or by means of suggesting decisions (false advice).

\section{Reflexive Management in Discourse Communities}

A reflexive game is probable only in a case where agents can reach performative equilibrium they can act concordantly at reflexion levels $n>0$. This condition is fulfilled in the case where there are mechanisms of intercommunication broadly agreed upon among people. These mechanisms have been preserved within an appropriate discourse community (Kommunikationsgemeinschaft) [1] shared by members with a suitable degree of discoursal expertise (i.e. members possess one or more genres in the communicative furtherance of its aims and know a specific lexis) and with a degree of relevant content to provide information and feedback.

Any discourse community represents a group of people who are in permanent interactions with each other and exchange performative propositions. This community is self-organised. Due to common discourse it can reach an informational equilibrium, and also a parity of creative reasoning as well as emotional consensus in interchanging performative propositions.

Members of a discourse community have a common speech competence (Sprachkompetenz) [1], sufficient for interactions. Let us recall that speech competence as such is comprehended neither by members of a discourse community, nor by outside agents, but its possession is a necessary condition for entry into an appropriate discourse community. Speech competence is understood as the knowledge and ability to use language in accordance with different contexts. Thus, modelling by speech competence is a key notion for managing a discourse community.

Any stable group of people united by joint interests is a discourse community. It is presented by appropriate forms of consolidation. In some cases the emotional community of its members leads to the appearance of corporate ethics, i.e. to sharing values and priorities. Systems of sanctions, such as blame or elimination from the group, are possible also. 
A discourse community can be transformed into an appropriate social institute. Then group interests are formulated in an explicit form. Common centres of decision making appear and individual acts of activity are coordinated with joint plans. Within social institutes the discourse community becomes a hierarchical system. Its degree of complexity is presented by the opacity of the decision-making mechanism (the closeness of the mechanism for how creative reasoning is constructed, the hiding of a maximal reflexion level of the centre). The different degrees of openness of a social structure is possible also.

A discourse community is a multiagent system whose participants have the ability to act, including a freedom to choose states and strategies of speech behaviour. Besides a possibility of choice of activity schemata, members of a discourse community bear characteristic interests and preferences which can contradict interests and preferences of other members.

In any multiagent system we assume that there is a collection of subjects and objects which are units of the system, but they can be different by the nature: rational, irrational, intelligent, phantom agents, etc. Among these items there is a family of informational, controlling and other links, including subordination relations and distributions of the right to make decisions.

Rules, according to which the criteria of interaction effectiveness (performative equilibrium) are made, define which agents are rational or irrational. The dynamics of a system depends on the variety of preferences of agents and on the ways of consensus in the context of control actions. The order of system functioning can be revealed by detecting a sequence of process data and a choice of strategies made by system members. Thus, the functioning order depends on how often different strategies are chosen.

The life engineering cycle has the following stages: (1) design (concept design, detailed design, validation), (2) realization (plan manufacturing, manufacture, test), service (sell and deliver, use, maintain and support, dispose). By analogy it is possible to point out a performative cycle of a discourse community: (1) showing joint interests, (2) definitions of appropriate forms of performative equilibriums to reach joint interests, (3) implementation and realizing corresponding performative equilibriums, (4) loss of joint interest. For example, the performative cycle of a club of salsa fans consists of the stages: acquaintance of several salsa fans, finding a place for regular meetings (for example, in a bar), realisation of meetings, acceptance of new members, closing.

In hierarchic discourse communities (for example, in social institutes) the dialogue with the centre can be considered a reflexive game. The more difficult the hierarchic multiagent system, the higher the order of reflexion of the centre. The game task consists in explicating performative cycles of the system in order to uncover the centre's mechanisms of planning and stimulation and, then, to involve the centre in a reflexive game, having the order of reflexion sufficient for obtaining victory in this reflexive game.

Centralised systems are a variety of hierarchic ones. Their disadvantage is in that subordinates ignore a part of their obligations and avoid full responsibility, meaning that their manager is completely responsible. The effectiveness of management for such a business is insignificant. The manager is too busy dealing with routine, and the employees half idle, expecting visit from the manager. In such systems any reflexive game with the centre has a low reflexion level, therefore performative equilibriums do not assume a high cognitive and emotional consensus of participants.

The system, in which there is a delegation of powers, where the decision-making process is distributed throughout the entire system of management, is more rational than the centralised system. The higher tasks of organisation are divided into many more detailed tasks for which solutions specific employees are responsible. Hence, each employee (1) surely knows what action (s)he is responsible for; (2) knows what resources (s)he can use independently and in what cases (s)he can ask the manager about additional resources; (3) knows how outcomes of activity are evaluated and knows the method of reward for success. These conditions provide the system with complex reflexive games making the system more stable performatively.

\section{References}


1. Apel, K.-O. Transformation der Philosophie 2. Das Apriori der Kommunikationsgemeinschaft, Berlin: Suhrkamp, 1999.

2. Brandenburger, A. Epistemic game theory: an overview, In St. Durlauf, L. Blume, (eds.), The New Palgrave Dictionary of Economics, $2^{\text {nd }}$ ed., Palgrave Macmillan, 2008.

3. Chkhartishvili, A. G. Bayes-Nash equilibrium: Infinite-depth point information structures, Automation and Remote Control, 64 (12), 2003, pp. 1922-1927.

4. Chkhartishvili, A. G. Concordant informational control, Automation and Remote Control, 73 (8), 2012, pp. 1401-1409.

5. Chkhartishvili, A. G. \& D. A. Novikov. Models of reflexive decision-making, Systems Science, 30 (2), 2004, pp. 45-59.

6. Chkhartishvili, A. G. Reflexive games: Transformation of awareness structure, Automation and Remote Control, 71 (6), 2010, pp. 1208-1216.

7. Frederick, S. Cognitive Reflexion and Decision Making, Journal of Economic Perspectives, 19 (4), 2005, pp. 24-42.

8. Lefebvre, V. A. The basic ideas of reexive game's logic, Problems of systems and structures research, 1965, pp. 73-79 (in Russian).

9. Lefebvre, V. A. Algebra of Conscience, Dordrecht: D. Reidel, 1982.

10. Lefebvre, V. A. Lectures on Reflexive Game Theory, Los Angeles: Leaf \& Oaks, 2010.

11. Schütz, A. \& T. Luckmann. Strukturen der Lebenswelt, Berlin: Suhrkamp, 1979.

12. Schumann, A. Payoff Cellular Automata and Reflexive Games, Journal of Cellular Automata, 9 (4), 2014, pp. 287-313.

13. Schumann, A. Probabilities on streams and reflexive games, Operations Research and Decisions, 1, 2014, pp. 71-96.

14. Schumann, A. Reflexive Games and Non-Archimedean Probabilities, p-Adic Numbers, Ultrametric Analysis and Applications, 6 (1), 2014, pp. 66-79.

15. Searle, J. R. Speech acts; an essay in the philosophy of language, Cambridge: Cambridge University Press, 1969.

16. Searle, J. R. \& D. Vanderveken. Foundations of Illocutionary Logic, Cambridge: Cambridge University Press, 1984. 